\title{
Design And Implementations Of Kindergarten Management System Based On Fingerprint Identification And RFID Technology
}

\author{
Fei Lao ${ }^{1}$ \\ ${ }^{1}$ Department of Information Engineering, Binzhou Vocational College, Binzhou, 256600, China
}

Keywords: fingerprint identification,RFID,database, wireless network, system design.

\begin{abstract}
The kindergarten management system has two parts:one is a kindergarten entrance guard system,the other part is Safety Management System.Aiming at the problem of Pick-up and Delivery children in kindergarten, it is difficult to identify the people who meet children, and there are some other problems, such as low efficiency, frequent accidents. Kindergarten Entrance Guard System based on fingerprint identification is designed, which not only effectively identify the pick-up person, also can effectively inspect the kindergarten staff attendance. The radio frequency identification technology is applied on strangers visiting and child safety in kindergarten. The Kindergarten Entrance Guard System consists of four parts, namely fingerprint verification module, fingerprint identification module, host computer and wireless network module. The Kindergarten Safety Management System consists of three parts, namely data label, reading equipment and control center.FPM10A was use to fingerprint identification module and nRF24LE1 chip has been used in data label and MSP430F149 singlechip has been used in reading equipment and CC2430 chip has been used in RF sending-receiving unit.Zigebee technology was employed in the wireless network, and host computer can work with Java and Tomcat while the database chooses SQL server2008.The experiment proved that system is rather reliable and to be promote the use of this system.
\end{abstract}

\section{Introduction}

Objects of Kindergarten enrollment are three-to-six year old children who are very active and lovely but lack of self protection consciousness. Recently, many malignant events about children in kindergarten happened. While we are immersed in sorrow, we should analysis of the causes of these accidents and find efficient measures to prevent such accidents. The cause of the accident is mainly attributed to two aspects, kindergarten and parents. For example the kindergarten did not carry out the effective methods to manage visitors and parents, pick-up cards lost from parents are found by lawbreakers, kindergarten system is not rigorous and normative, infant pick-up card can be easily forged, the kindergarten teachers do not have effective identification the offenders.

With the development of science and technology, information technology, network technology,the fingerprint recognition technology and RFID technology has been rapidly developed. Fingerprint identification technology has been applied in many industries, which no longer only applied to criminal investigation in the past. For example, Zhao Zhe put the application of fingerprint identification technology in vehicle anti-theft, Liang Jing has applied the fingerprint identification into the attendance of university student, Bai Yan presents the application in the randomly selected seat of examination etc. RFID technology has been widely applied in all aspects of life, such as radio frequency technology will be applied to the logistics industry, the radio frequency technology used in the patient's identification, RFID technology will be applied to books and the radio frequency technology applications in car theft. Therefore, in this paper we combine fingerprint recognition technology with RFID technology to apply on kindergarten management. Through analysis and compare, we find these two technologies are low cost, convenient, high accuracy and fast response speed, which can avoid many disadvantages on infant pick-up card brings, therefore these two technologies application in kindergarten can effectively improve work efficiency, reduce the teachers the pressure of work, avoid a lot of accidents, and provide a safe and comfortable living environment for children. The RFID system composition block diagram is as the 
figure1.

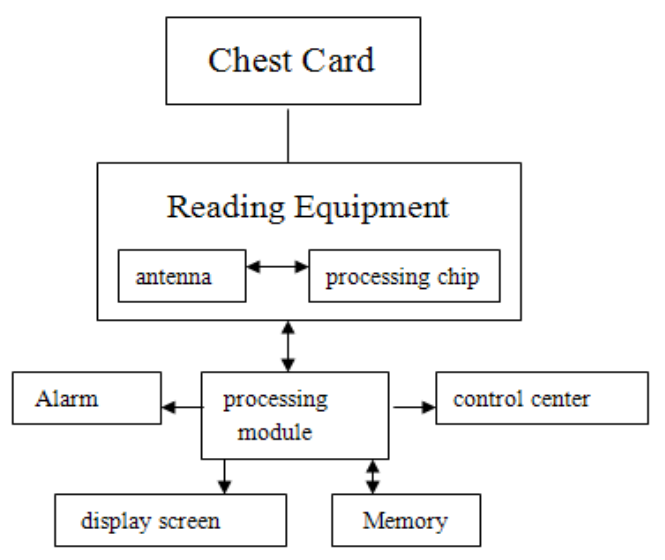

Fig.1:RFID system composition block diagram

\section{The System Workflow And Structure}

\section{fingerprint recognition workflow}

There are no two exact fingerprints in the world, and fingerprints have relative stability in a longer period of time, using the above features of fingerprint to design kindergarten entrance guard system based on fingerprint identification.

Mainly realizing the functions of two parts, one is the identification of enter kindergarten personnel, at the same time inspecting attendance of the kindergarten staff. Another is checking identification and recording relevant information of the children's parents. The system comprises a fingerprint acquisition module, a fingerprint identification module, wireless network module and host computer module.

Working process of this system is as follows. Firstly, to kindergarten teachers, administrative personnel, their fingerprints are gathered and taken sample and save these data into the database. Then fingerprints of children and their parent also should be gathered and taken sample and save these data into the database. Staffs of kindergarten need to pass the fingerprint verification to enter the kindergarten. In the meantime the arrival time of staff would be stored in the database, thus completing the attendance management. Parents should take fingerprint verification to enter the gate of kindergarten when they send their children in the early morning. After handing over their children to teachers, children conduct fingerprint verification again at the gate of classroom. Then this information will store in the database, which is convenient for management. At the school hours, parents need to firstly pass the fingerprint verification at the gate of nursery, and then enter the kindergarten, and inspected by fingerprint verification again to withdraw a child from school. The parents' information and children's information will be displayed in the screen of class computer, and these data will be stored in database. Finally, the parents would be given the right to take their children away.

The process of fingerprint identification is as follows. Firstly, using the fingerprint acquisition equipment collects the fingerprint. Next, the captured fingerprint image will be processed with Binary method in order to obtain the high quality image of fingerprint. To be more effective to compare the fingerprint, employing the technology of coordinate positioning obtains the overall and specific characteristics of fingerprint feature and store these data.

Then entering the step of fingerprint matching, the fingerprint will be matched the between required identified fingerprint and exist fingerprint. If the match is successful, parents can successfully enter kindergarten. If the fingerprint matching is fail, the system will prompt again for fingerprint recognition, if fail again, access control system will send alarm. 


\section{RFID workflow}

The principles of wireless radio frequency identification are as follows, RFID technology can quickly realize the non-contact, and correct goods identification. RFID technology is composed of three parts, respectively the data labels, reading device and the corresponding processing software. The data labels and the reading devices inside embed antenna and processing chip. The type of work is mainly divided into two categories, one category is reading devices after the switch is turned on, will take the initiative to capture the read range data label information, data labels to the RF signal reading device is sent after the reception, will work through the induction current data labels. In addition, a class is a data tag chip started first, and then the RF signal and sends corresponding signal acquisition equipment, read in their own work scope, identify. Information collection, reading device transmits the information to be translated, and then processing software to send information back to the corresponding translation of information processing. Children, teachers are responsible to help the wearer by embedding data label chest mark. According to the characteristics of children, chest mark in the production was used with non-toxic, tasteless material, and chest mark should possess the characteristics of bright color, easy to wear, long service life etc.. In the embedded chip chest mark stored in an information children's names, photos, class, the teacher, parents, parents contact name. In order to ensure the child's information can be timely, effective, correct being monitored, the data labels should be choose the wireless RF chip distance corresponding.

Radio frequency identification work process is as follows, the kindergarten sets children activity security zone, once the children beyond the safety area, for example, go to the nursery door, the system immediately issued a warning, children at present location information and details of the children will also notice to the kindergarten entrance guard, the kindergarten teachers and kindergarten relevant responsible person, thus effectively to avoid the occurrence of children lost by lawbreakers away and other accidents. In the kindergarten may occur key point set wireless radio frequency reading device, such as a kindergarten kitchen, boiling water, power supply and so on are real, the roof may pose a threat for the baby's safety, once the child into the key points, read the device immediately issued a warning, and the children at the present location and detailed information to inform the relevant personnel, avoid children danger. Temporary visitors in the past the doorman allowed after wearing temporary access card, can enter the kindergarten, and according to the visitors set path to visit, once the visitor beyond the scheduled path, start the alarm immediately, and notify the kindergarten security personnel, so as to further ensure that the baby's safety. Visitors at the end of kindergarten after the visit, to ease the guard office dear will wear the access card to access immediately cancelled.

\section{Design of the system hardware}

\section{Hardware design of kindergarten entrance guard system}

In all of the hardware equipment, only fingerprint collection needs $5 \mathrm{~V}$ voltage, and the others are the need for 3.3V, thus adding NCP1117ST33 into the power circuit, which could the change voltage successfully.

The fingerprint identification module is the core part of the whole system. The quality of fingerprint acquisition and fingerprint identification affect the accuracy of the whole system. After analysis and comparison, this system use FPM10A. FPM10A is composed of two parts, a fingerprint sensor and DSP.

Wireless network is mainly made up of four parts, the access node, two types of send-receive node and router and coordinator. In fact, major components of the access node and send-receive node are CC2530. In addition, power supply, the fingerprint acquisition module and alarm are equipped with the above nodes.

\section{Hardware design of kindergarten safety system}

The safety of children in kindergarten and supervision to visit in temporary personnel need to use radio frequency technology, hardware design of the module is mainly related to the data labels, reading device, reading device specific information contained in the data read labels, and the control 
center handles the information given to the computer for processing.

According to the current kindergarten scale, the most distant reading device and data labels will be set to 100 meters, that is to say, in this range, reading device can accurately collect data corresponding to labels, finally the band for the $2.4 \mathrm{GHz}$ band. According to the selected frequency band of the data labels and the function, the chip nRF24LE1 is considered in the system design. The chip integrates wireless transmission system, enhanced MCU and multi-function peripherals. Low price, powerful function, low power consumption is the main reason for choosing this chip. Because the chip power consumption is low, so the selected button battery as the power supply of $3 \mathrm{~V}$. Chest mark for the data label, in order to guarantee the normal work of data labels, tags in the data are arranged to remind function, once the chest mark is not working properly, will immediately start red warning lights.

Read device hardware includes a central processing unit, RF transceiver unit and other units.The central processing unit selects a low power MCU MSP430F149, in the whole equipment plays the role of duct. Responsible for scanning the work according to the management orders, processing control of the whole information read operation is completed at the same time misreading information; complete decoding data read label information; information exchange center control and complete computer, the memory is capable of storing the children's name, gender, teachers and other detailed information. MSP430F149 microcontroller provides the port can provide interrupt, synchronous communication and other functions. In order to test the chip is working correctly, the SCM is also provided with a timer. In order to meet the demand of serial communication, microcontroller must have a serial communication module.

Radio frequency transceiver unit is a bridge of communication between the central processing unit and a data label. The core of RF transceiver unit adopts CC2430, the chip is a powerful, 3.3V power filter can meet the power requirements, RF transceiver unit to ensure the correctness and reliability of the signal transceiver.

\section{Design of system software}

Kindergarten Entrance Guard System (KEGS) can be run on the Windows XP and the windows7 operating system. As the JAVA language has the characteristics of stability and safety, and also a multi-threaded object-oriented language. JAVA is the sublimation of $\mathrm{c}^{++}$language, developing many merits of $\mathrm{C}++$, at the same time also abandoning many of its defects. Therefore JAVA is chosen to develop system, Eclipses as development platform. As the advantages of open source, good stability and other advantages, Tomcat is chosen as the web application server.

To system structure, the popular B/S and C/S structure are compared. The B/S structure which is especially suitable for LAN and the World Wide Web is the evolution and upgrading of the structure of the $\mathrm{C} / \mathrm{S}$. The user normal employ browser to login and perform. Most of things are processed by server-side. This system structure is low cost, convenient use, and security of database can be effectively guaranteed. Therefore, B/S structure finally is selected.The B/S system frame diagram is as the figure 2 .

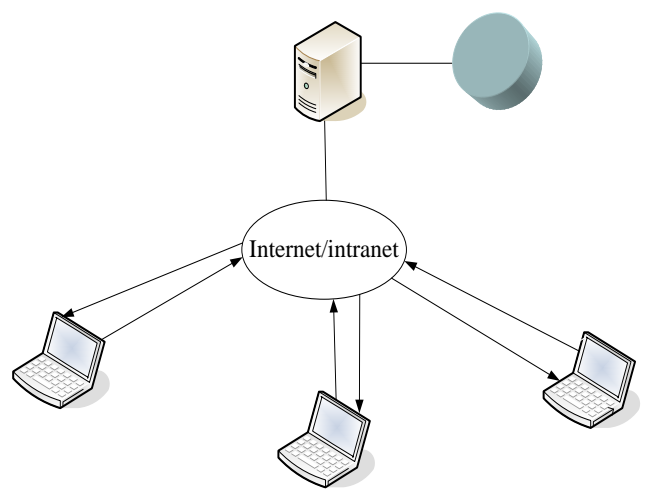

Fig.2: B/S system frame diagram

The coordinator plays a crucial role in the system, which receive the data from bottom, and 
analyze these data, at the same time can perform the upper command, transmits the data to the end nodes. In the system due to the choice of the Java language, so the serial communication needs to use the Java class library. Interface design, parameter design, and monitoring different controls are designed by different classes. Fingerprint software which is used to collect the fingerprints of kindergarten staff, children, and pick-up person's convert data into a fingerprint pattern, and then these data can be stored in database as standard fingerprints. The new collected fingerprint should be compared with the standard fingerprint, to verify the identity of the true and false.This process mainly includes fingerprint image, detailed information, instruction receiving and sending area, and serial port name and baud rate.

The database serves a key function in the whole system, whose performance decides the performance of whole system. After careful analysis, SQL Server 2008 was selected for the safety, reliability and expansibility reasons. The design of Database is strictly comply with standards and procedures. To begin with, for the each part of database, data flow diagram was completed according to different treatment process. In the stage of conceptual design of the database, local E-R diagram can be completed firstly, global E-R map is finished secondly, finally, optimize the whole system. In order to reduce the data redundancy phenomenon in the database, in the course of database design, paradigm will be converted to BCNF paradigm. The database includes user table, children table and fingerprint table, staff attendance record and so forth.

The user table mainly stores the username and password of management staff and the teacher in charge of a class. Users can search their children's shuttle information and attendance rate according to their own specific circumstances. This table includes the fields of user's ID, user's name, password and user type. Fingerprint table is mainly used for storing the fingerprint information, including the objects of fingerprint acquisition, more specifically, kindergarten staff, children and their two parents. Major fields include fingerprint ID, name, identity, acquisition time, class, finger etc. the role of kindergarten shuttle table is storage the information of transfer time, pick-up persons, non-registered staff shuttle information, which includes children number, children's name, class, pictures of children, name of their parents, leaving time, delay time and explanation of special case. Employee attendance table is used to store the kindergarten staff with detailed information. This table is used to record attendance information of the kindergarten staff. Staff attendance information includes employee ID, name, gender, attendance time, leaving time.

\section{Conclusions}

Safety problem in the kindergarten has already become the focus of the society. Kindergarten Management System Based On Fingerprint Identification And RFID Technology, the fingerprint identification technology and RFID technology are combined to effectively solve the problem of kindergarten attendance of staff. More important, the people who enter into nursery can be strictly controlled. Those who pick up and send children can be accurately and effectively identified, avoiding false and wrong pick-up accident.Wear chest card to avoid the hurt due to dangerous accident and it is worth using widely.

\section{Acknowledgements}

The research work was supported by the Project of Binzhou Science and Technology Development Plans under Grant No. 2011ZC0402.

\section{References}

[1]HONG L. Automatic personal identification using fingerprints. USA: Michigan State University, 1998(1):25-36

[2] Nils Petter Gregersen. Systematic cooperation between driving schools and parents in driver education, an experiment. Accident Analysis \& Prevention, 1994, 26(4): 453-461 
[3] Anders Nyberg, Nils Petter Gregersen, Mats Wiklund. Practicing in relation to the outcome of the driving test. Accident Analysis \& Prevention, 2007, 39(1): 159-168

[4] E.Newham.The Biometric Report.New York:SJB Services,1995:11-20P

[5] Dinesh P Mital and Eam Khwang Teoh, An Automated Matching Technique for Fingerprint Identification, 1997 First International Conference on Knowlwdge-Based Intelligent Electronic Systems, 21-23 May1997.

[6] Outemzabet, S.; Nerguizian, C.,"Accuracy Enhancement of an Indoor ANN-based Fingerprinting Location System using Kalman filtering", The 19th IEEE International Symposium on Personal, Indoor and Mobile Radio Communications, 15-18 Sept.2008, pp. 2-4.

[7] Zhang Weimei. Study about IOT's Application in Digital Agriculture Construction[C].IEEE. 2011. pp:2578-2581.

[8] International Telecommunication Union. The Internet of Things[R]. ITU Internet Reports, 2005.

[9] European Commission. Internet of Things in 2020, Roadmap for The Future[R]. Joint European Commission/EPoSS, May 27, 2008.

[10] Hankima Chaouchi. The Internet of Things-Connecting Objects to the Web[M]. British Library Ctaloguing-in-Publication Data. 2010. 